\title{
Secreted Salt Increases Reaumuria Soongarica's Ability to Compete in Grassland Areas
}

changshun wang ( $\square$ wcs2001000@126.com )

h University https://orcid.org/0000-0002-8032-9038

huiqing wang

h. univercity

wei wang

h. univercity

cunzhu liang

h. univercity

huamin liu

h.univercty

lixin wang

h. univercity

\section{Research article}

Keywords: Reaumuria soongarica, stress tolerance, interspecies competition, tradeoff, soil conductivity $\mathbb{Z}$ Inner Mongolia

Posted Date: August 9th, 2021

DOl: https://doi.org/10.21203/rs.3.rs-789822/v1

License: (c) (i) This work is licensed under a Creative Commons Attribution 4.0 International License.

Read Full License 


\section{Abstract}

Background: The aim of this study was to identify and explore the community formation mechanism of $R$. soongarica in the eastern Mongolian Plateau grassland. The experimental site was located in an ancient lake basin with saline soil in a desert steppe.

Results: Soil conductivity of $R$. soongarica was significantly higher than that of the two herbs, S. glareosa and $A$. polyrhizum, at all soil depths $(\mathrm{P} \leq 0.001)$. The daily salt secretion rate ranged from $1 \%$ to $2 \%$ of the fresh leaf weight in the different communities and increased with increased soil conductivity. With increased canopy size of $R$. soongarica, the distance between the shrubs and herbs also increased. The correlation between the $R$. soongarica canopy diameter and the distance to the nearest $S$. glareosa $\left(R^{2}=\right.$ $0.4065 ; P<0.05)$ was higher than that to the nearest $A$. polyrhizum $\left(R^{2}=0.1256 ; P<0.05\right)$. The growth of the three species was not salt-dependent; however, $R$. soongarica was significantly more salt-tolerant than the two herbs. The two herbs significantly limited the growth of $R$. soongarica seedlings at low soil conductivity $(\leq 600 \mu \mathrm{S} / \mathrm{cm})$, but not at high soil conductivity $(\geq 1000 \mu \mathrm{S} / \mathrm{cm})$.

Conclusions: Salt secretion by $R$. soongarica leaves results in the formation of a "saline island," which leads to soil conductivity increasing significantly under the canopy of $R$. soongarica. This increase in soil conductivity of the saline island effectively reduces the interspecies competition advantage of the two herbs. This highlights the competitiveness of $R$. soongarica in salt-stressed environments and facilitates the establishment of this desert shrub in saline regions on the desert steppe.

\section{Background}

Species establishment and community stability are two sides of the same coin (Godsoe et al. 2015; Richardson and Pyšek 2006; Seastedt and Pyšek 2011). In nature, zonal plant communities alternate along environmental gradients (Scherrer et al. 2017; Ungar 1998) and have clear and stable boundaries (Michalet et al., 2014; Sizykh 2007). The typical desert plant Reaumuria soongarica has a unique distribution; $R$. soongarica not only dominates desert communities in Asia, but also forms communities in the eastern Mongolian Plateau grassland where soils are generally saline. Therefore, $R$. soongarica has managed to adapt to the extreme drought of the desert environment and can also occupy areas with high moisture, where other plants dominate with different strategies across vegetation zones (Kefu et al. 2002). Generally, a zonally dominant species cannot spread to other vegetation zones (Ungar 1998). This raises the question: what is the mechanism by which $R$. soongarica breaks this general rule?

In reality, some plant species cannot adapt to environmental stress, whereas others that have adapt to a stressed environment cannot spread to areas with greater precipitation or higher temperature regimes (Mao et al., 2018; Ungar 1998). For example, the desert steppe prevents the establishment of desert plant seedlings via strong interspecific competition imposed by its dominant populations, which maintains the relative stability of the vegetation distribution (Hooper and Dukes 2010; Naeem and Wright 2003). Conversely, steppe plants have difficulty adapting to harsh desert habitats and cannot effectively survive 
in the desert (Austrheim and Eriksson 2003). This phenomenon is the result of the combined effects of plant physiological tolerance to abiotic stress and interspecies competition (i.e., the ability of a plant to inhibit the growth of another plant) (Herron et al. 2007; Kenkel et al. 1991). It remains unclear how the environmental tolerance and interspecies competition of plants act on establishment of $R$. soongarica in saline regions within the steppe. Our study aimed to answer this question by focusing on the adaptability of $R$. soongarica to stress and interspecies competition in a grassland community.

The leaves of $R$. soongarica can secrete salt onto the ground (Xiaoning et al. 2019), which is similar to Mesembryanthemum crystallinum that can absorb salt from deeper soil layers and deposit the salt on the soil surface, leading to the death of neighboring, less salt-tolerant plants, and ultimately forming a community (Brockie et al. 1988). Owing to the limited tolerance of a given plant to its environment, this interspecies competition advantage is not identical across environments (Muthukrishnan et al. 2018; Douda et al. 2018). Therefore, the first hypothesis of the present study is that whether the soil salinity under $R$. soongarica is higher than that of herbs, and whether the presence of $R$. soongarica affects the position of the herb.

The tolerance of plants to extreme environments are based on specialized functions (Szabados et al. 2011; Botella et al. 2005). In a stress-less environment, these special functions are redundant and may become a burden on interspecific competition (Qi et al. 2018; Margit 2012). Thus, plants with higher tolerance will show a reduced ability in terms of access to resources under interspecies competition in a stress-free environment (Seastedt and Pyšek 2011; Liancourt et al. 2005; Scholes and Archer 1997; Ungar 1996). We assumed that $R$. soongarica was unable to occupy areas with better environmental conditions (i.e., vast grassland areas, not just salty areas) because it cannot compete with local dominant species, especially herbs. Therefore, the second hypothesis of the present study is that $R$. soongarica is unable to compete with dominant plants in grassland areas under conditions of low soil salinity.

\section{Results}

\section{Soil conductivity for the different plant species}

With an increase in soil depth, the soil conductivity for the three species was increased. The soil conductivity of $R$. soongarica was significantly higher than that of the two herbs, $S$. glareosa and $A$. polyrhizum, at all soil depths ( $P \leq 0.001)$ (Fig. 2). The difference in soil conductivity between the shrubs and herbs increased with the increase in total soil conductivity. Soil conductivity was significantly higher in $A$. polyrhizum than that in $S$. glareosa $(P \leq 0.001)$, especially in the $R$. soongarica $+S$. glareosa community that had low soil conductivity (Fig. 2a).

\section{Salt secretion rate of $\mathrm{R}$. soongarica}

The daily salt secretion rate ranged from $1-2 \%$ of the fresh leaf weight in the different communities. The amount of excreted salt increased over time (Fig. 3). There were significant differences in salt secretion 
rates among the three communities $(P<0.01)$. The salt secretion rate increased with an increase in soil conductivity.

\section{Population Distribution Patterns In The Community}

With an increase in the canopy size of $R$. soongarica, the herbs were situated further away from the shrub species. The correlation between the $R$. soongarica canopy diameter and the distance to the nearest $S$. glareosa $\left(R^{2}=0.4065 ; P<0.05\right)$ was higher than that to the nearest $A$. polyrhizum $\left(R^{2}=0.1256 ; P<0.05\right)$ (Fig. 4).

\section{Effect Of Salinity On Plant Growth}

Soil conductivity had a negative effect on the growth of the three species (Fig. 5a). The relative biomass of the three species decreased with an increase in the soil conductivity, indicating that these species were not salt-dependent. Compared with the two herbs, $R$. soongarica had a significantly higher salt tolerance $(P<0.001, N=54)$, as its relative biomass decreased more slowly than that of the herbs at a conductance of 200 or $600 \mu \mathrm{S} / \mathrm{cm}$. The relative biomass of the two herbs at $600 \mu \mathrm{S} / \mathrm{cm}$ was decreased to less than $50 \%$, whereas that of $R$. soongarica was decreased to $80 \%$. The relative biomass of the shrub was decreased to $50 \%$ at $2000 \mu \mathrm{S} / \mathrm{cm}$, whereas that of the two herbs fell almost to zero under the same conditions. At low soil conductivity (200 or $600 \mu \mathrm{S} / \mathrm{cm}$ ), the relative biomass of $S$. glareosa decreased slightly faster than that of $A$. polyrhizum; therefore, A. polyrhizum was more salt-tolerant than S. glareosa.

Effect of interspecies competition on the growth of R. soongarica

The relative biomass of $R$. soongarica when planted with either of the two herbs, S. glareosa and $A$. polyrhizum, was significantly lower than when planted on its own at soil conductivity values of 0,200 , and $600 \mu \mathrm{S} / \mathrm{cm}(P<0.001$; Fig.. 5b). However, the relative biomass of $R$. soongarica was not significantly different among the single $R$. soongarica and mixed cultures at soil conductance exceeding $1000 \mu \mathrm{S} / \mathrm{cm}$. The herbs significantly limited the growth of $R$. soongarica seedlings at low soil conductivity $(\leq 600$ $\mu \mathrm{S} / \mathrm{cm}$ ); however, their effect was eliminated at higher soil conductivity ( $\geq 1000 \mu \mathrm{S} / \mathrm{cm}$ ), which was detrimental to the herbs.

\section{Discussion}

\section{Competitiveness and environment}

Processes related to special functions can alter the local environment. Thus, the limited competitiveness of the original vegetation (Chaneton et al. 2012; Vilà and Weiner 2004) is important for the successful establishment of plant species in the community (Seastedt and Pyšek 2011; Richardson and Pyšek 2006; Brown and Archer 1999; White and Currie 1983). Our results showed that the conductivity of every soil 
layer was significantly higher for $R$. soongarica than for the two herbs (Fig. 2) and the higher the soil conductivity, the higher the salt secretion by $R$. soongarica (Figs. 1 and 3 ). Therefore, the physiological process of salt secretion by $R$. soongarica leaves created soil salt spatial heterogeneity in the coexisting community which was important for its establishment in the grassland.

A plant can inhibit the growth of its competitors by developing special physiological functions that can increase its ability to access resources (Rathcke and Lacey 1985). The competitiveness of the species will then be greater than that of the neighboring species (Golivets and Wallin 2018). The higher salt content under $R$. soongarica was named "saline islands". The conductivity of the soil in the saline island was significantly increased with higher soil salinity (Fig. 2a and b). Because the amount of salt secretion was related to the dry weight of the leaves (Fig. 3), it is easy to understand why more salt was secreted when the $R$. soongarica canopy was larger. This promoted the growth of saline islands and was perhaps the reason why the herbs were found to be positioned further away from the shrub as the canopy area of $R$. soongarica increased (Fig. 4). This was especially highlighted by the distribution of herbs with different salt tolerances. In our community tests, the distance between $R$. soongarica and $S$. glareosa was larger than that between $R$. soongarica and A. polyrhizum (Fig. 4) because of the lower salt tolerance of S. glareosa (Fig. 5a).

Plants can gain higher competitiveness through allelopathy (Fynn et al. 2005), which increases their chances of establishment (Qi et al. 2018). This phenomenon is not always the result of a specialized physiological behavior. For example, oak leaves can inhibit the growth of herbaceous plants (Facelli 1994) and the removal of alpine forest litter can considerably promote the growth of herbs (Wilson and Zammit 1992). Thus, the ability to change the environment (i.e. allelopathy) is key point for the survival of plants in this community; it changes the level of interspecies competition and ultimately leads to changes in the community structure. Our results supports first hypothesis that salt secretion by $R$. soongarica leaves changes the soil salinity and thereby the distribution of plants because of the different salt tolerances.

\section{Competitiveness and tolerance}

The decreased biomass of the three species at higher soil conductivity in the laboratory showed that the three species preferred environments that were free of salt stress (Fig. 5a) and that a saline environment was not physiologically required for their optimal growth. Additionally, salinity limited the growth of the herbaceous plants substantially more than that of $R$. soongarica. Therefore, $R$. soongarica has a physiological preference for the non-saline soils of the desert steppe in eastern Inner Mongolia. Many drought- or salt-tolerant species grow better in a stress-free environment (Chaneton et al. 2012; Ashton and Lerdau, 2007; Kenkel et al. 1991); however, the realized niche of most stress-tolerant plants often deviates from their fundamental niche because of interspecies competition (Herron et al. 2007).

There was a reversal in competitiveness in our laboratory experiment. At lower salt conductivity, the high competition level of the herbs inhibited the growth of the $R$. soongarica seedlings (Fig. 5b). This 
competition from locally dominant herbs explains why $R$. soongarica has not been established widely on the desert steppe in eastern Inner Mongolia (i.e., because of its low soil salinity) (Kefu et al. 2002). Thus, as proposed in the second hypothesis, $R$. soongarica can adapt to drought and salinity; however, its seedlings have lost the ability to compete with the dominant herbs in a salt-free environment. A species with both high tolerance and interspecies competitiveness will compete with, and eventually eliminate, other species, leading to reduced species diversity in a community. However, this is not the result of longterm evolution. Rather, there are tradeoffs made between different adaptabilities of the plants (e.g., tolerance and interspecies competitiveness or acquisitive and conservative) (Diaz et al. 2004; Vilà and Weiner 2004; Crawley 1990). An exclusive relationship between environmental tolerance and competitive ability to resources (Kenkel et al. 1991) has been demonstrated experimentally by many studies (Chaneton et al. 2012; Mazía et al. 2001). At low soil nutrient levels, species with high tolerance are more successful. Conversely, when the soil nutrient level is high, the same species are at a competitive disadvantage (Berendse 1994; Aerts and Vanderpeijl 1993). Environmental pressure limits the competitiveness of species with strong resource access capabilities and, in turn, increases the competitiveness of highly tolerant species (Liancourt et al. 2010). Reaumuria soongarica has evolved salt glands as an adaptation to drought in desert regions or high-salinity steppes. However, this specialized trait and capability provides no advantage in the absence of drought or salt stress and the plant becomes powerless when competing with dominant grassland species that do not have these specializations (Ayres 2008), especially during the seedling period.

Successful species establishment is easy to understand based on the assumption that the competitiveness of the species distributed across zones is greater than that of the native species (Seastedt and Pyšek 2011; Vilà and Weiner 2004). However, the long-term presence of the vegetation zone proves that this assumption is not universally true (Theoharides and Dukes 2007). Another explanation is that competitiveness is not "real," but is a process related to special functions (Williamson and Fitter 1996). Our results support the notion that the ecological mechanisms of $R$. soongarica establishment, which rely on salt secretion, facilitate the spread of $R$. soongarica across vegetation zones and the formation of a community on the desert steppe in eastern Inner Mongolia. The soil conductivity of the saline islands (Fig. 2) was high enough to inhibit herb growth (Fig. 5a) and $R$. soongarica growing on a saline island could resist competition from the herbs and maintain its growth environment (Fig. 5b). Reaumuria soongarica takes full advantage of its specialized traits and abilities, from "weak" to "strong," whereby it becomes established on the desert steppe. Our results add new knowledge to the existing literature, and thus help to advance our understanding of how some shrub species with strong environmental tolerance can survive in desert steppes and establish a stable community. The interspecies competition advantage of a species is inverse to its ability to tolerate abiotic stress, indicating a tradeoff among different abilities, which determines the distribution of plants.

\section{Conclusion}

Salt secreted by $R$. soongarica affects the soil conductivity environment and forms saline islands, which effectively inhibit the growth of other dominant herbs, and thus increase the competitiveness of $R$. 
soongarica. Reaumuria soongarica is a highly salt-tolerant species whose growth does not depend on soil salt. Our results showed that the growth of seedlings in areas with low soil conductivity was significantly limited by herbs; however, this limitation disappeared at high soil conductivity because of the low salt tolerance of the herbs. Our data reveal the phenomenon and mechanisms of interspecific competition of $R$. soongarica via its allelopathic effect involving inorganic salts, which is the reason for the establishment of $R$. soongarica in saline areas of a desert steppe.

\section{Methods}

\section{Experimental location}

In the grasslands of Inner Mongolia, $R$. soongarica communities are found in scattered patches. An ancient lake basin containing an $R$. soongarica community, situated east of Erlianhot City, Inner Mongolia, China $\left(43^{\circ} 23^{\prime} 16^{\prime \prime}-43^{\circ} 42^{\prime} 28^{\prime \prime} \mathrm{N}, 112^{\circ} 01^{\prime} 1^{\prime \prime}-112^{\prime} 01^{\prime} 78^{\prime \prime} \mathrm{E} ; 910 \mathrm{~m}\right.$ above sea level), was selected as the experimental site. The diameter of the ancient lake basin is approximately $15 \mathrm{~km}$ and the depth is $30 \mathrm{~m}$. The soil conductivity (soil salinity) increases with decreasing elevation, which produces clear boundaries between the plant communities at the site (Fig. 1). Outside the ancient lake basin is the zonal plant community, which is dominated by Stipa glareosa. From the basin margin to the center, the plant communities are $R$. soongarica $+S$. glareosa, $R$. soongarica + Allium polyrhizum, pure $R$. soongarica, $R$. soongarica + Kalidium foliatum, and pure K. foliatum.

The mean monthly air temperature ranges from $-18.1^{\circ} \mathrm{C}$ in January to $23.3^{\circ} \mathrm{C}$ in July, with a mean annual temperature of $3.4^{\circ} \mathrm{C}$. The mean annual precipitation (1971-2000) is $142.2 \mathrm{~mm}, 67 \%$ of which falls from June to August. The surface soil is composed of $66 \%$ sand, $20 \%$ silt, and $14 \%$ clay, with a mean pH of 7.1 outside the ancient lake basin. These natural plant communities are not fenced because they are used for grazing by domesticated sheep and camels. Grazing is the main land use method, and the intensity of grazing is relatively light.

We have the permission to collect the plant samples by the local government. The voucher specimen with the collect number(Wang 20080705) was identified by professor Liqing Wang and deposited in HIMC.

\section{Experimental Design}

\section{Soil conductivity}

There are four types of $R$. soongarica communities in the experimental area: $R$. soongarica $+S$. glareosa, $R$. soongarica $+A$. polyrhizum, pure $R$. soongarica, and $R$. soongarica $+K$. foliatum. Reaumuria soongarica coexists with the two herbs only-in the $R$. soongarica $+S$. glareosa and $R$. soongarica $+A$. polyrhizum communities. In order to understand the difference in soil conductivity (soil salinity) with different types of plant individuals in this coexistence community of herbs and shrubs, soil samples were collected from each of the three species in the $R$. soongarica $+S$. glareosa and $R$. soongarica $+A$. 
polyrhizum communities at depths of $0-5 \mathrm{~cm}, 5-15 \mathrm{~cm}, 15-25 \mathrm{~cm}, 25-35 \mathrm{~cm}, 35-45 \mathrm{~cm}$ and $45-55$ $\mathrm{cm}$. Each sampling was performed in triplicate. The total of soil samples number was108. Soil conductivity was measured using the conductance method (Multi 340i; WTW Xylem Analytics, Weilheim, Germany). The pure $R$. soongarica and $R$. soongarica $+K$. foliatum communities were not selected because they contained no herbs.

\section{Salt secretion rate}

To understand the differences in the salt secretion rate of $R$. soongarica with different soil conductivity in the different communities, 12 samples of the $R$. soongarica individuals were chosen randomly from the $R$. soongarica $+S$. glareosa, $R$. soongarica $+A$. polyrhizum, and pure $R$. soongarica communities. The selected individuals were divided into four groups (four $\times$ three). One branch per individual was randomly marked. The accumulated salt on the leaf surface of the selected branches was washed repeatedly with distilled water at the beginning of the experiment to ensure that the surfaces of the leaves were salt-free. The salt secretion rate was measured for three individuals on the first, third, fifth, and seventh days. The marked branches were soaked in $150 \mathrm{~mL}$ of distilled water to measure the conductance of the liquid using a conductivity meter (Multi 340i; WTW Xylem Analytics) and were removed from the stem to measure the weight of the fresh leaves using an electronic balance (YP1002N; INESA, Shanghai, China) at the end of the experiment. The leached water was collected in containers to prevent it from falling to the soil surface. The measurement of the conductance of the liquid was undertaken in July and the entire experiment was repeated in August 2009. Although the experiment was performed twice, owing to the destruction of some plants by animals, we only obtained two and three complete datasets in July and August, respectively.

\section{Population distribution patterns}

The amount of salt secretion was found to correlate positively with the crown size of $R$. soongarica. The salt secreted by the $R$. soongarica leaves was likely to increase the soil conductivity, and thus affect the growth of the herbs in the community. This could be demonstrated by the canopy area of $R$. soongarica and the distance between $R$. soongarica plants and the neighboring herbs. To investigate the effect of the salt secretion of the shrub on the herbs, the canopy diameter and distance to the nearest $S$. glareosa and $A$. polyrhizum plants were measured for 30 samples randomly selected $R$. soongarica individuals in the $R$. soongarica $+A$. polyrhizum community using a measuring tape. The $R$. soongarica $+S$. glareosa community was not selected because $A$. polyrhizum was rarely found in this community. The crown is assumed to be a perfect circle, and its area is calculated from the diameter.

\section{Laboratory cultivation experiment}

To verify the change in the competitiveness of different plants under different salt conditions, we conducted cultivation experiments in the laboratory. Reaumuria soongarica, S. glareosa, and $A$. polyrhizum seeds were collected in August 2008. In March 2009, the seeds were soaked in distilled water for $30 \mathrm{~min}$ and treated with $0.1 \%$ gibberellin to break the seed dormancy. The seeds were then placed between double filter papers and germinated in a dark environment in a Petri dish. The germinated seeds 
were transferred to a paper cup ( $12 \mathrm{~cm}$ in height, $7 \mathrm{~cm}$ top diameter, and $5 \mathrm{~cm}$ bottom diameter) that was filled with $300 \mathrm{~g}$ of river sand, which had been rinsed repeatedly with distilled water to remove any salt and nutrients from the soil, thus simulating quartz sand.

Based on the abundance of the three species observed in their natural communities, the ratio of herbs to shrubs was 6:1. Five combinations of three species were prepared: one combination consisted of six seedlings of $S$. glareosa arranged in a ring $1 \mathrm{~cm}$ from the cup wall, the second combination was similarly prepared but with six A. polyrhizum seedlings, and the third combination consisted of only one $R$. soongarica seedling placed in the center of the cup. In the mixed species combinations, six S. glareosa or A. polyrhizum seedlings were placed around one $R$. soongarica seedling in a cup.

Six NaCl salinity levels were prepared: $0,200,600,1000,1500$, and $2000 \mu \mathrm{S} / \mathrm{cm}$ (soil conductivity). This salinity gradient was based on the soil conductivity measured in different natural communities. Each treatment was prepared in triplicate.

Based on local precipitation and soil nutrient levels, $5 \mathrm{~mL}$ of Hoagland nutrient solution was applied every 2 weeks and $10 \mathrm{~mL}$ of distilled water was added each time and four times (once per week) to each cup. The experiment lasted for 12 weeks. During the fifth week of the experiment, different amounts of $\mathrm{NaCl}$ $(0,0.18,0.54,0.9,1.35$, and $1.8 \mathrm{~g})$ were added to each cup to obtain six salinity levels. The seedlings were cultivated in an artificial climate chamber (ZRX-1000 ESW; TESTMART, Hangzhou, China) at $25^{\circ} \mathrm{C}$ and $70 \%$ relative humidity with a $14 \mathrm{~h} / 10 \mathrm{~h}$ light/dark regime.

At the end of the experiment, the entire plant was dried and weighed. The relative biomass of the three species in each treatment was calculated based on the biomass of the plant cultivated individually at 0 $\mu \mathrm{S} / \mathrm{cm}$ soil conductivity as a control.

\section{Statistical analysis}

To test the difference in soil conductivity of the different plant individuals in this coexistence community of herbs and shrubs and the difference in the salt secretion rate of $R$. soongarica with different soil conductivity (in different communities), one-way analysis of variance (ANOVA) was used at every soil layer and each sampling time. Pearson's correlation coefficient, which was used to characterize the population distribution patterns, was calculated for the distance between one of the two herbs $(S$. glareosa and A. polyrhizum) and $R$. soongarica and canopy diameter. ANOVA was used to test the effect of salt and competition on the three species. When significant salt or competition effects were observed, Duncan's post-hoc tests were used to assess the differences among the species. Statistical tests were performed using SPSS version 15.0 for Windows (SPSS Inc., Chicago, IL, USA).

Material statement

This manuscript comply with institutional, national, or international guidelines and the Convention on the Trade in Endangered Species of Wild Fauna and Flora. 


\section{Declarations}

\section{Ethics approval and consent to participate}

Not applicable

\section{Consent for publication}

Not applicable

\section{Availability of data and materials}

All data generated or analysed during this study are included in this published article. Reaumuria soongarica is not a rare plant for protection. We have the permission to collect the plant samples by the local government. The voucher specimen with the collect number(Wang 20080705) was identified by professor Liqing Wang and deposited in HIMC.

\section{Competing interests}

The authors declare that they have no competing interests

\section{Funding}

This work was supported by the National Natural Science Foundation of China (31700410, 31560146); the Program for Young Talents of Science and Technology in the Universities of Inner Mongolia Autonomous Region (NJYT-18-B27); a Science and Technology Major Project of Inner Mongolia (ZDZX2018054) and the Science and Technology Project of Inner Mongolia (201802100). The funding body had no role in the design of the study and collection, analysis, and interpretation of data and in writing the manuscript.

\section{Authors' contributions}

$\mathrm{CW}$ analyzed and interpreted the data . H W was a major contributor in writing the manuscript. WW, $\mathrm{CL}$, $\mathrm{HL}$, LW gave detailed instructions to the manuscript. All authors read and approved the final manuscript.

\section{Acknowledgments}

We thank Xin zhang and Jian Hou for English language editing. We are grateful to the Associate Editor and referes for providing valuable comments.

\section{References}

1. Aerts R, Peijl MJVD. A Simple Model to Explain the Dominance of Low-Productive Perennials in Nutrient-Poor Habitats. Oikos. 1993;66:144.. 
2. Ashton IW, Lerdau MT. Tolerance to herbivory, and not resistance, may explain differential success of invasive, naturalized, and native North American temperate vines. Diversity and Distributions. 2007;14:169-78.

3. Austrheim G, Eriksson O. Recruitment and life-history traits of sparse plant species in subalpine grasslands. Canadian Journal of Botany. 2003;81:171-82.

4. Ayres P G. the Darwins at the dawn of plant science. The aliveness of plants: the Darwins at the dawn of plant science. Pickering \& Chatto. 2008.

5. Berendse F. Competition between Plant Populations at Low and High Nutrient Supplies. Oikos. 1994;71:253.

6. Botella MA, Rosado A, Bressan RA, Hasegawa PM. Plant Adaptive Responses to Salinity Stress. Plant Abiotic Stress. 2005,37-70.

7. Brockie R, Loope LL, Usher MB, Hamann O. Biological invasions of island nature reserves. Biological Conservation. 1988;44:9-36.

8. Brown JR, Archer S. Shrub Invasion Of Grassland: Recruitment Is Continuous And Not Regulated By Herbaceous Biomass Or Density. Ecology. 1999;80:2385-96.

9. Chaneton EJ, Mazía N, Batista WB, Rolhauser AG, Ghersa CM. Woody Plant Invasions in Pampa Grasslands: A Biogeographical and Community Assembly Perspective. Ecotones Between Forest and Grassland. 2012;:115-44.

10. Crawley M J. The population dynamics of plants. Philosophical Transactions of the Royal Society of London. Series B: Biological Sciences. 1990;330:125-40.

11. Csipkés $M$. The examination of the profitability and competitiveness regarding the energy plantations of woody plants in the region of Észak-Alföld. Applied Studies in Agribusiness and Commerce. 2012;6:5-11.

12. Diaz S, Hodgson J, Thompson K, Cabido M, Cornelissen J, Jalili A, et al. The plant traits that drive ecosystems: Evidence from three continents. Journal of Vegetation Science. 2004;15:295-304.

13. Douda J, Doudová J, Hulík J, Havrdová A, Boublík K. Reduced competition enhances community temporal stability under conditions of increasing environmental stress. Ecology. 2018;99:2207-16.

14. Facelli JM. Multiple Indirect Effects of Plant Litter Affect the Establishment of Woody Seedlings in Old Fields. Ecology. 1994;75:1727-35.

15. Fynn R, Morris C, Kirkman K. Plant strategies and trait trade-offs influence trends in competitive ability along gradients of soil fertility and disturbance. Journal of Ecology. 2005;93:384-94.

16. Godsoe W, Murray R, Plank MJ. The effect of competition on species distributions depends on coexistence, rather than scale alone. Ecography. 2015;38:1071-9.

17. Golivets M, Wallin KF. Neighbour tolerance, not suppression, provides competitive advantage to nonnative plants. Ecology Letters. 2018;21:745-59.

18. Herron PM, Martine CT, Latimer AM, Leicht-Young SA. Invasive plants and their ecological strategies: prediction and explanation of woody plant invasion in New England. Diversity and Distributions. 
2007;13:633-44.

19. Hooper DU, Dukes JS. Functional composition controls invasion success in a California serpentine grassland. Journal of Ecology. 2010;98:764-77.

20. Kefu Z, Hai F, Ungar I. Survey of halophyte species in China. Plant Science. 2002;163:491-8.

21. Kenkel NC, Mcilraith AL, Burchill CA, Jones G. Competition and the response of three plant species to a salinity gradient. Canadian Journal of Botany. 1991;69:2497-502.

22. Liancourt P, Callaway RM, Michalet R. Stress Tolerance And Competitive-Response Ability Determine The Outcome Of Biotic Interactions. Ecology. 2005;86:1611-8.

23. Mao W, Zhao X, Sun Z, Felton AJ, Zhang T, Li Y, et al. Limiting similarity mediates plant community niche hypervolume across a desert-steppe ecotone of Inner Mongolia. Environmental and Experimental Botany. 2018;153:320-6.

24. Mazia NC, Chaneton EJ, Ghersa CM, León RJ. Limits to tree species invasion in pampean grassland and forest plant communities. Oecologia. 2001;128:594-602.

25. Michalet R, Maalouf J-P, Choler P, Clément B, Rosebery D, Royer J-M, et al. Competition, facilitation and environmental severity shape the relationship between local and regional species richness in plant communities. Ecography. 2014;38:335-45.

26. Muthukrishnan R, Hansel-Welch N, Larkin DJ. Environmental filtering and competitive exclusion drive biodiversity-invasibility relationships in shallow lake plant communities. Journal of Ecology. 2018;106:2058-70.

27. Naeem S, Wright JP. Disentangling biodiversity effects on ecosystem functioning: deriving solutions to a seemingly insurmountable problem. Ecology Letters. 2003;6:567-79.

28. Qi M, Sun T, Xue S, Yang W, Shao D, Martínez-López J. Competitive ability, stress tolerance and plant interactions along stress gradients. Ecology. 2018;99:848-57.

29. Rathcke B, Lacey EP. Phenological Patterns of Terrestrial Plants. Annual Review of Ecology and Systematics. 1985;16:179-214.

30. Richardson DM, Pyšek P. Plant invasions: merging the concepts of species invasiveness and community invasibility. Progress in Physical Geography: Earth and Environment. 2006;30:409-31.

31. Scherrer D, Massy S, Meier S, Vittoz P, Guisan A. Assessing and predicting shifts in mountain forest composition across 25 years of climate change. Diversity and Distributions. 2017;23:517-28.

32. Scholes RJ, Archer SR. Tree-Grass Interactions In Savannas. Annual Review of Ecology and Systematics. 1997;28:517-44.

33. Seastedt T, Pyšek P. Mechanisms of Plant Invasions of North American and European Grasslands. Annual Review of Ecology, Evolution, and Systematics. 2011;42:133-53.

34. Sizykh AP. Plant communities of environmental interfaces as a problem of ecology and biogeography. Biology Bulletin. 2007;34:292-6.

35. Szabados L, Kovács H, Zilberstein A, \& Bouchereau A. Chapter 4 - Plants in extreme environments: Importance of protective compounds in stress tolerance. Advances in Botanical Research, 2011;57: 
105-150.

36. Theoharides KA, Dukes JS. Plant invasion across space and time: factors affecting nonindigenous species success during four stages of invasion. New Phytologist. 2007;176:256-73.

37. Ungar IA. Effect of Salinity on Seed Germination, Growth, and Ion Accumulation of Atriplex patula (Chenopodiaceae). American Journal of Botany. 1996;83:604.

38. Ungar IA. Are biotic factors significant in influencing the distribution of halophytes in saline habitats? The Botanical Review. 1998;64:176-99.

39. Vilà $M$, Weiner $J$. Are invasive plant species better competitors than native plant species? - evidence from pair-wise experiments. Oikos. 2004;105:229-38.

40. White RS, Currie PO. Prescribed Burning in the Northern Great Plains: Yield and Cover Responses of 3 Forage Species in the Mixed Grass Prairie. Journal of Range Management. 1983;36:179.

41. Williamson M, Fitter A. The Varying Success of Invaders. Ecology. 1996;77:1661-6.

42. Wilson SD, Zammit CA. Tree litter and the lower limits of subalpine herbs and grasses in the Brindabella Range, ACT. Austral Ecology. 1992;17:321-7.

43. Xiaoning N, Minxia L, Gong Z, Sujuan X, Quandi LI, \& Xiaoxuan J. Spatial distribution pattern and association of reaumuria soongarica and caragana roborovskyi in central loess plateau, Chinese Journal of Ecology. 2019,38(2):354-360.

\section{Figures}

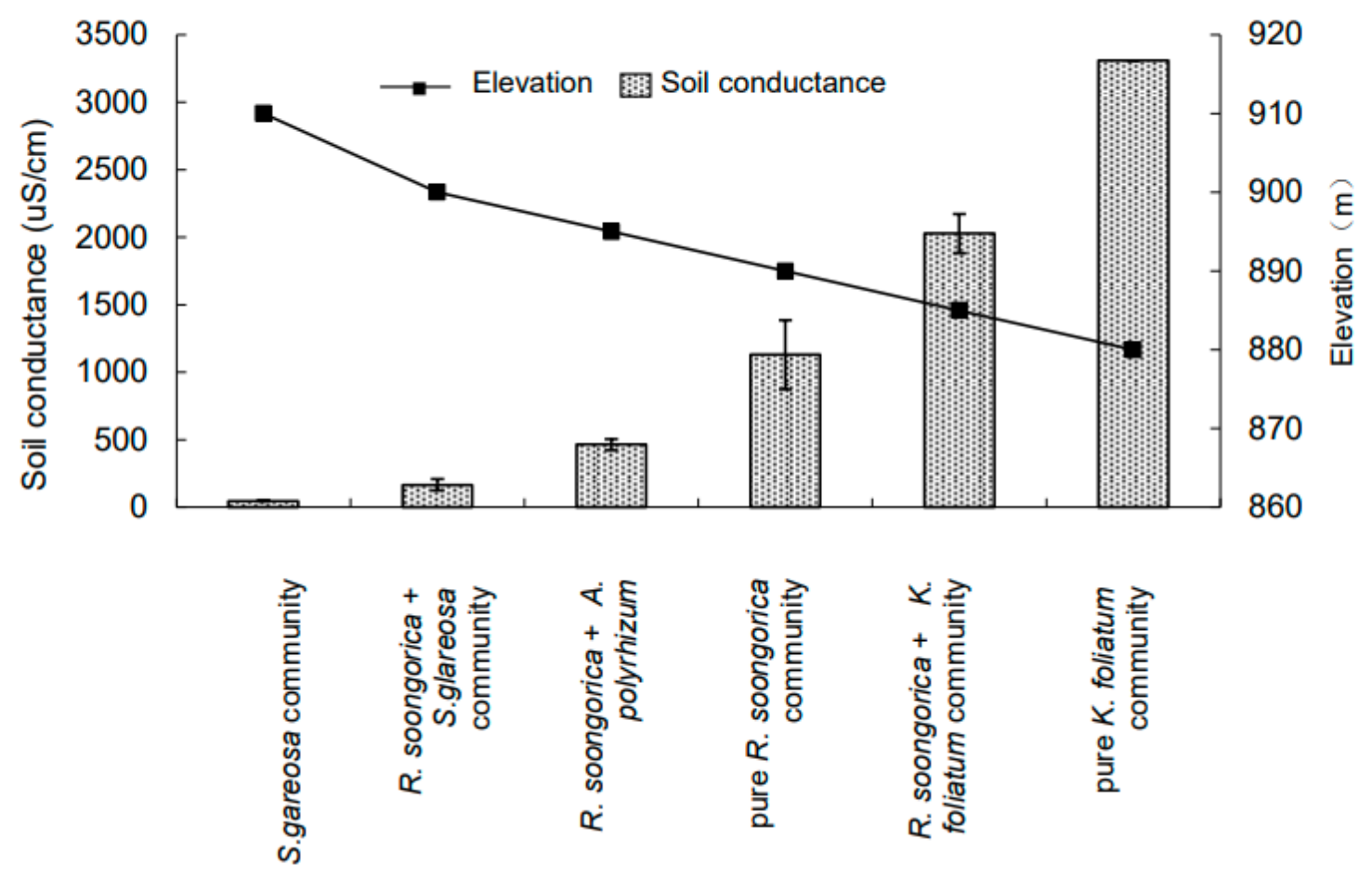


Figure 1

Mean soil conductivity $(0-55 \mathrm{~cm})$ and elevation in different communities at the research site. The vertical lines at the top of the bars represent the standard deviation $(\mathrm{N}=3)$.

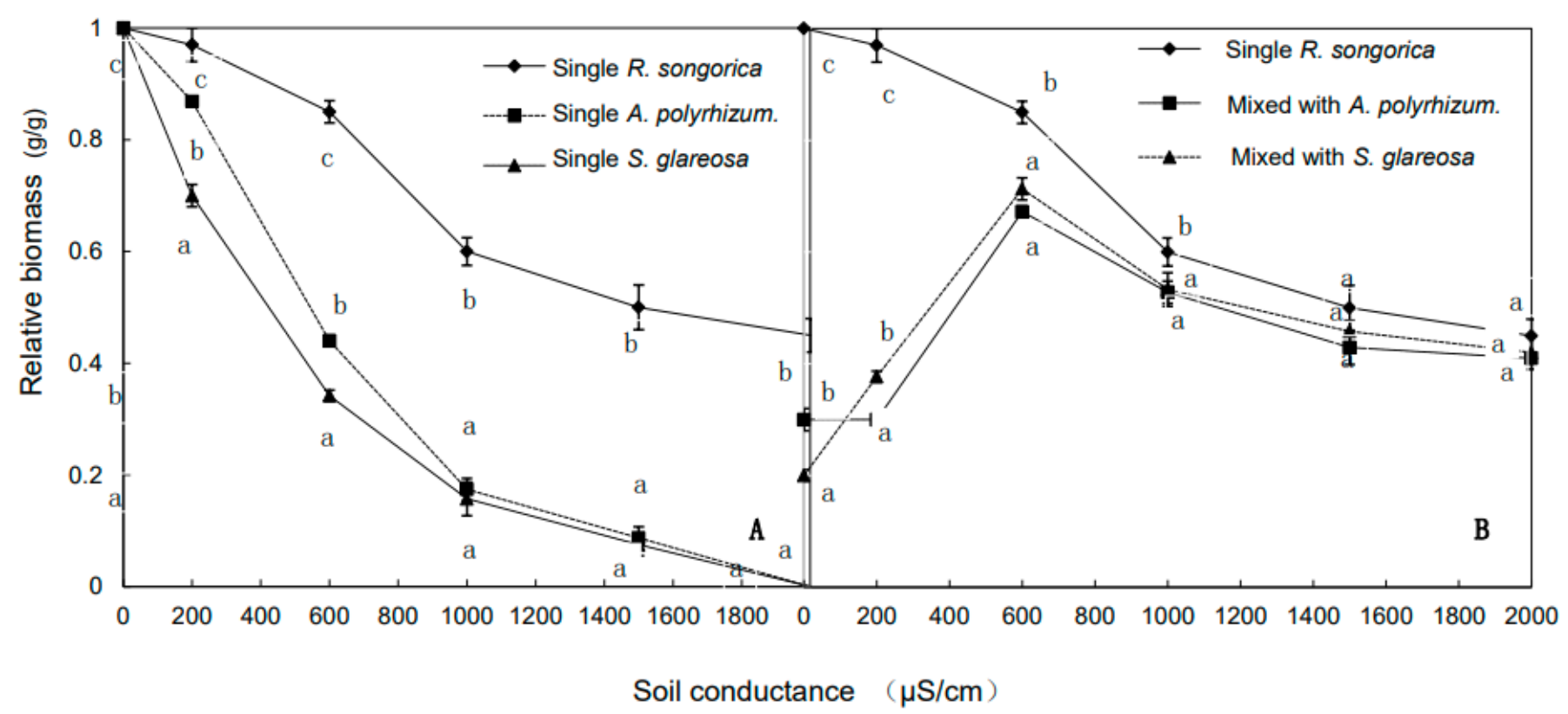

\section{Figure 2}

Soil salt conductivity under the three species (Reaumuria soongarica, Stipa glareosa, and Allium polyrhizum) in the R. soongarica + S. glareosa community (a) and the R. soongarica + A. polyrhizum community (b) at different soil depths (mean \pm standard error; $N=9$ ). Uppercase letters indicate significant differences among the three species at different soil depths according to Duncan's test $(\mathrm{p}<$ 0.05). 


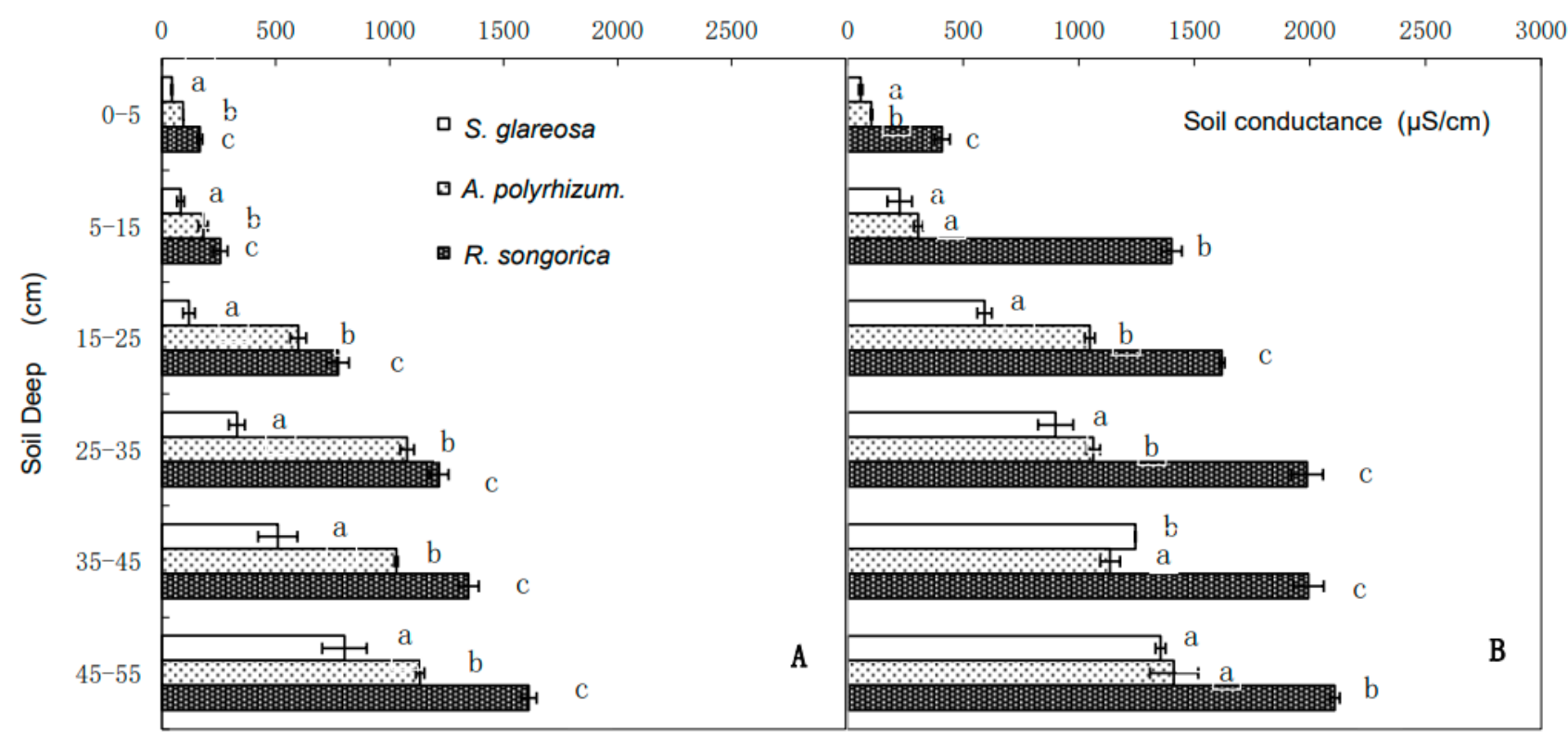

\section{Figure 3}

Quantitative analysis of salt excreted by R. soongarica in different communities (R. soongarica + S. glareosa, R. soongarica + A. polyrhizum, and pure R. soongarica) with different soil conductivities at two different times (mean \pm standard error; $N=9$ ). Uppercase letters indicate significant differences among the communities on a different day according to Duncan's test $(p<0.05)$.

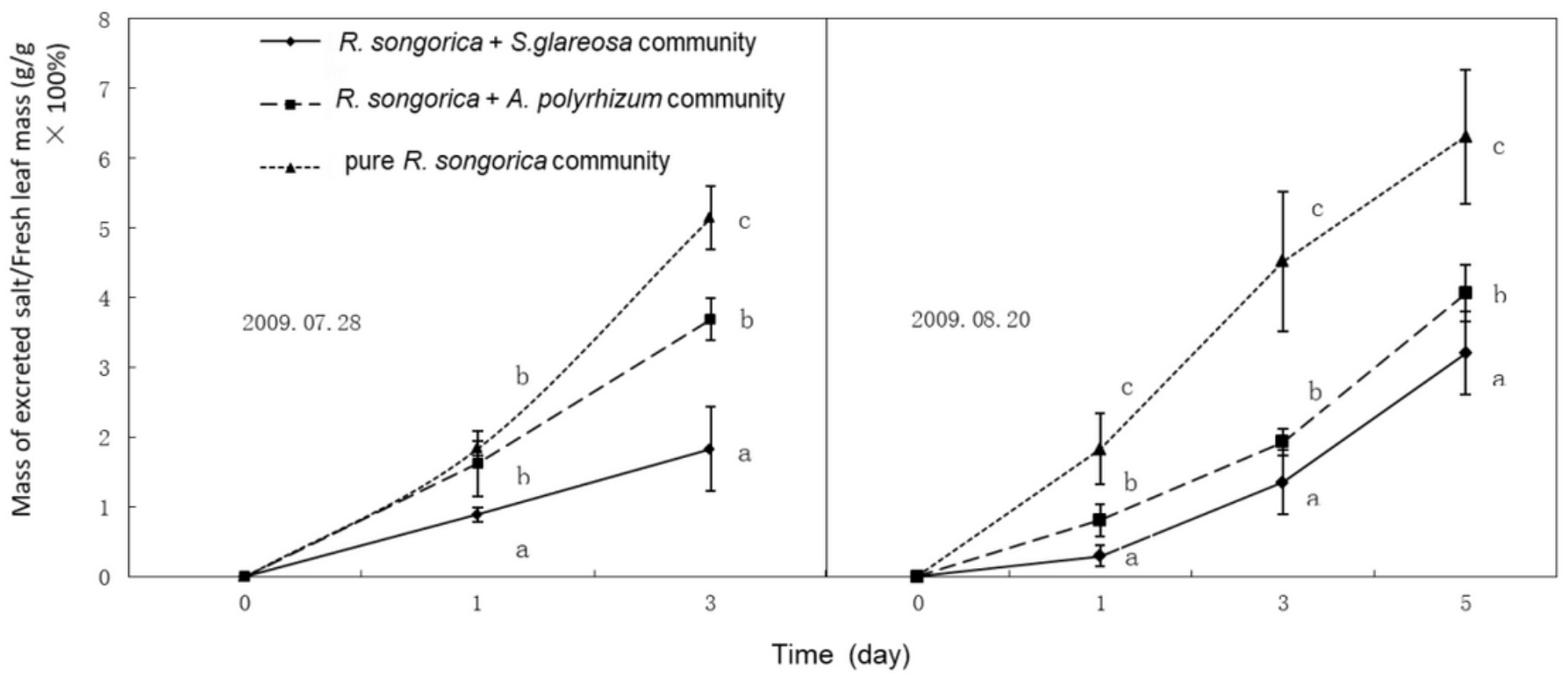

Figure 4

Correlation between Reaumuria soongarica canopy size and its closest distance to Stipa glareosa (a) and Allium polyrhizum (b). 


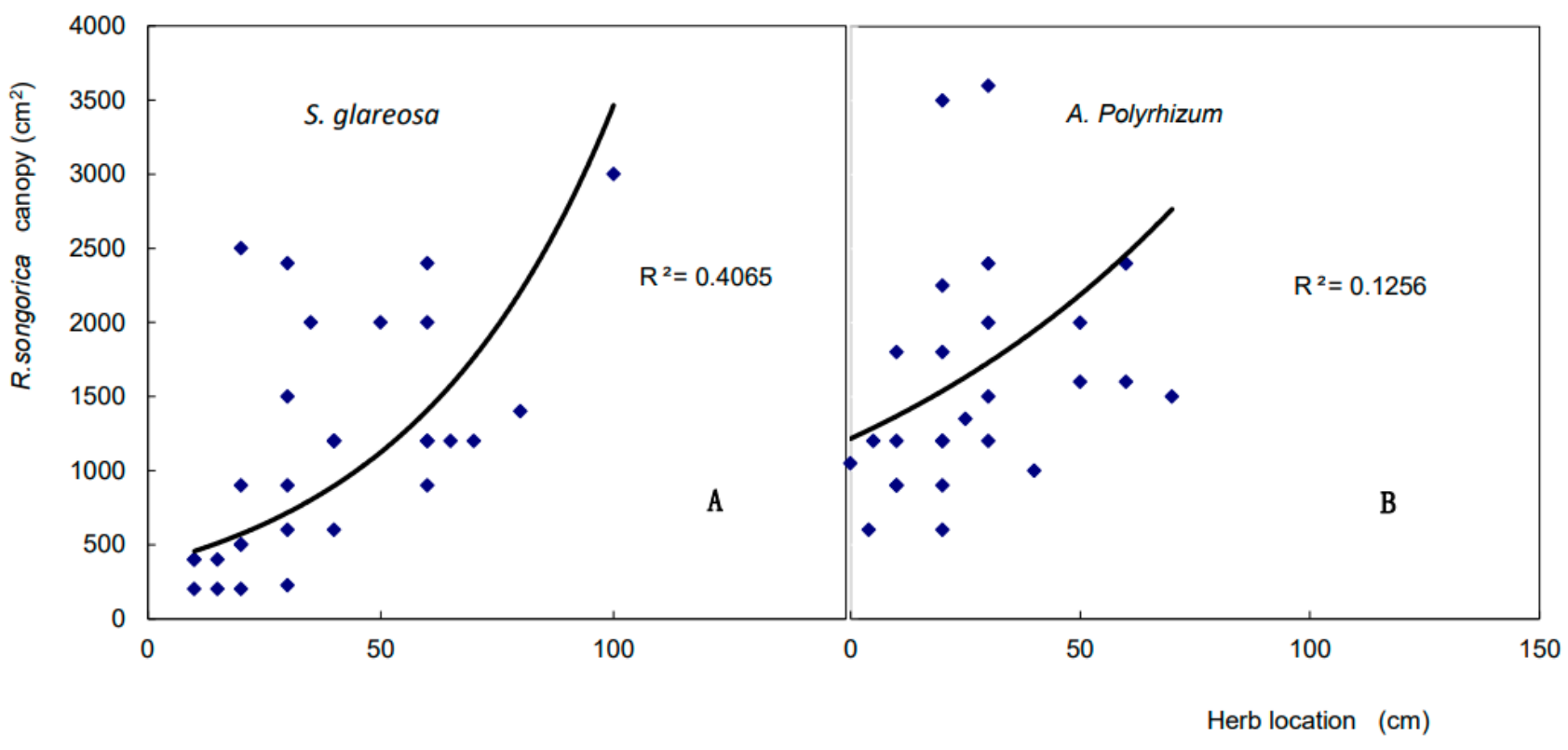

Figure 5

Relative biomass of the three species (Reaumuria soongarica, Stipa glareosa, and Allium polyrhizum) cultivated separately (a), and of R. soongarica cultivated individually or with herbs (b). Values are means \pm standard error $(\mathrm{N}=3)$. Uppercase letters indicate significant differences among different conditions according to Duncan's test $(p<0.05)$. 